\title{
Geminin, Ki67, and minichromosome maintenance 2 in gastric hyperplastic polyps, adenomas, and intestinal-type carcinomas: pathobiological significance
}

\author{
Kohei Shomori ${ }^{1}$, Keisuke Nishihara $^{1}$, Takayuki Tamura ${ }^{1}$, Shigeru Tatebe ${ }^{2}$, Yasushi Horie ${ }^{3}$, Kanae Nosaka ${ }^{1}$, \\ Tomohiro Haruki ${ }^{1}$, Yuki Hamamoto ${ }^{1}$, Tatsushi Shiomi ${ }^{1}$, Motoki Nakabayashi ${ }^{1}$, and Hisao Ito \\ ${ }^{1}$ Division of Organ Pathology, Department of Microbiology and Pathology, Faculty of Medicine, Tottori University, 86 Nishi-cho, Yonago, \\ Tottori 683-8503, Japan \\ ${ }^{2}$ Division of Surgical Oncology, Department of Surgery, Faculty of Medicine, Tottori University, Yonago, Tottori , Japan \\ ${ }^{3}$ Department of Pathology, Tottori University Hospital, Yonago, Tottori, Japan
}

\begin{abstract}
Background. Geminin negatively regulates $\mathrm{Cdt1}$ and induces the formation of prereplicative complexes by loading minichromosome maintenance proteins $(\mathrm{Mcm})$ onto chromatin and limiting DNA replication to once per cell cycle. Recent studies have suggested that geminin expression is a marker of the S/G2/M phase of the cell cycle and is associated with a poor prognosis in various human malignancies. This study aimed to clarify the pathobiological role of geminin in intestinal-type gastric carcinoma, and its relationships with minichromosome maintenance $2(\mathrm{Mcm} 2)$ and Ki67 expression.

Methods. We performed western blot analysis of seven human gastric cancer cell lines, and immunohistochemical analysis of $\mathbf{7 2}$ gastric mucosal lesions and 128 surgically removed advanced intestinal-type gastric carcinomas. Double-labeling immunofluorescence was performed to identify the coexpression of geminin and Ki67.

Results. Geminin was detected in all cell lines. Geminin labeling indices (LIs) in hyperplastic polyps, low-grade adenomas, high-grade adenomas, and intestinal-type adenocarcinomas were $3.9 \%, 10.5 \%, 18.6 \%$, and $27.2 \%$, respectively. The equivalent LIs for Ki67 and Mcm2 were 17.7\%, 42.2\%, 52.6\%, and $59.7 \%$; and $26.7 \%, 70.0 \%, 67.8 \%$, and $77.8 \%$, respectively. Double-labeling immunofluorescence revealed coexpression of geminin and Ki67 in both normal and tumor cells. The LI for geminin was significantly correlated with $\mathbf{N}$ stage, International Union Against Cancer (UICC) stage, Mcm2 LI, and Ki67 LI. Patients in stages I-IV and stage III with higher LIs for geminin $(>25 \%)$ had significantly worse prognoses $(P<$ 0.05 and $P<0.04$, respectively). Univariate Cox regression analysis indicated that the overall survival of stage I-IV tumors was significantly correlated with high geminin LIs (relative risk $[R R]=1.94 ; P=0.04)$.

Conclusions. Geminin expression might reflect the biological nature of gastric intramucosal neoplasms and could be a possible prognostic marker in advanced intestinal-type gastric carcinomas.
\end{abstract}

Offprint requests to: K. Shomori

Received: August 6, 2009 / Accepted: May 12, 2010
Key words Geminin · Intestinal-type - Gastric carcinoma • Adenoma $\cdot \mathrm{Ki67} \cdot \mathrm{Mcm}$

\section{Introduction}

Gastric cancer is the world's second leading cause of cancer mortality after lung cancer, with an estimated 933000 new cases and 699000 deaths per year [1]. Growing evidence suggests that chemotherapy can improve disease-free intervals and overall survival $[2,3]$. Further studies are needed to identify molecular markers to help improve patients' prognoses.

Hyperplastic polyps (HPPs) and tubular adenomas are proliferative lesions of the gastric mucosa. Lowgrade adenomas (LGAs) are tubular structures lined by enlarged columnar cells with homogeneously blue pseudostratified nuclei in the superficial portion of the dysplastic tubules. High-grade adenomas (HGAs) are tubular structures with architectural distortion and pseudostratified cigar-shaped nuclei throughout the epithelium [4]. High-grade adenoma (HGA) is often difficult to differentiate from adenocarcinoma (ACA) [5-7]. LGA and HGA form a morphological spectrum with ACA. Considerable overlap currently exists between the histopathological diagnostic criteria for LGA, HGA, and ACA [8]. The possible subclassification of gastric mucosal proliferative lesions on the basis of their cell kinetics could aid in their differential diagnosis and offer new ways of predicting lesional growth rates.

Geminin is a $25-\mathrm{kDa}$ nuclear protein with a DNA replication-inhibitory function [9]. Through negative regulation of $\mathrm{Cdt} 1$, geminin induces the formation of prereplication complexes by loading minichromosome maintenance proteins $(\mathrm{Mcm})$ onto chromatin [10]. DNA polymerase binds to this complex and initiates DNA 
synthesis in $\mathrm{S}$ phase. This process repeats and limits DNA replication to once per cell cycle. To maintain this process, the level of geminin fluctuates throughout the cell cycle, being almost absent in G1 phase, but high in $\mathrm{S}$ and $\mathrm{G} 2$ phases and in the initial stage of $\mathrm{M}$ phase [11]. Geminin is thus responsible not only for regulation of the cell cycle, but also for the genomic integrity of cells. Geminin has been suggested to have tumor-suppressive functions, but previous reports have demonstrated that its expression was correlated with increasing tumor grade [12-15], and with poor prognosis in breast cancer patients [16]. Thus, discrepancies exist between geminin molecular function and the outcomes of clinical studies.

No study has examined the association between geminin expression and cell kinetics in gastric tumors and their prognostic significance in advanced gastric cancers. The aim of this study was to determine whether expression of the replication-inhibitory protein, geminin, could be useful for evaluating tumor proliferative potential and predicting outcome in patients with gastric carcinomas. We also aimed to clarify the relationships between the expression of geminin and the expression of minichromosome maintenance $2(\mathrm{Mcm} 2)$ and Ki67.

\section{Patients, materials, and methods}

\section{Cell culture and immunoblotting}

Human gastric cancer cell lines TMK-1 (poorly differentiated adenocarcinoma; PDA), MKN-1 (adenosquamous carcinoma), MKN-7 (well-differentiated adenocarcinoma; WDA), MKN-45 (PDA), MKN-74 (WDA), KATO III (signet-ring cell carcinoma), and HSC-39 (PDA) were routinely cultured in RPMI 1640 (Nissui Pharmaceutical, Tokyo, Japan) supplemented with $10 \%$ fetal bovine serum (JRH Biosciences, Lenexa, KS, USA) and $100 \mathrm{U} / \mathrm{ml}$ penicillin-streptomycin-L-glutamine (Wako Pure Chemical Industries, Osaka, Japan) at $37^{\circ} \mathrm{C}$ in a $5 \% \mathrm{CO}_{2}$ atmosphere. Cells were harvested for protein extraction as they reached subconfluence. Each $50 \mu \mathrm{g}$ of proteins was electrophoresed on sodium dodecyl sulfate-polyacrylamide gels, and transferred to polyvinylidene fluoride membranes. The membranes were incubated with primary antibodies at the following dilutions: rabbit anti-geminin antibody (1: 500; Santa Cruz Biotechnology, Santa Cruz, CA, USA) and mouse anti- $\beta$-actin antibody (1: 2000; Sigma-Aldrich, St. Louis, MO, USA). The membranes were then incubated with horseradish peroxidase-conjugated anti-rabbit $\operatorname{IgG}$ (1: 2000; MBL, Nagoya, Japan) and anti-mouse IgG (1: 2000; MBL). Immunoconjugates were detected by enhanced chemiluminescence (Amersham Pharmacia Biotech, Piscataway, NJ, USA).

\section{Patients and tissue specimens}

Seventy-two patients underwent endoscopic submucosal dissection (ESD) of gastric mucosal proliferative lesions. The lesions included 14 HPPs, 22 LGAs, 10 HGAs, and 26 ACAs. All but three depressed HGA lesions formed grossly flat or elevated lesions.

A total of 128 intestinal-type gastric carcinomas were selected on the basis of the cancer invasion being deeper than the muscularis propria (International Union Against Cancer [UICC]; T2-T4). Intestinal-type gastric carcinomas were defined as distinct glandular lumina often accompanied by papillary fold formation or solid components with distinct epithelial cords, as described in Lauren's classification [17]. All patients underwent radical en-bloc gastrectomy. No patients received preoperative radiochemotherapy. The median age was 70.0 years (range, 48-89 years). The mean follow-up period was 33.6 months, with a maximum of 161 months. There were 100 male and 28 female patients. Slides of each tumor were reviewed by pathologists with no knowledge of the clinical outcome, who assigned a pathological diagnosis according to the TNM staging system [4], the Japanese classification of gastric carcinoma [18], and the Lauren classification [17]. Details of the tumor series and clinicopathological parameters were obtained from clinical records. The study was approved by the Local Research Ethics Committee of Tottori University, Faculty of Medicine (No.283).

\section{Immunohistochemistry}

Representative tissue blocks containing a sample of peripheral tumor were selected for each patient. Formalin-fixed, paraffin-embedded tissues were cut onto silane-coated slides at $4-\mu \mathrm{m}$ thickness. The slides were dewaxed in xylene and rehydrated through graded alcohols. For antigen retrieval, the sections for Ki67 were incubated in $0.01 \%(\mathrm{w} / \mathrm{v})$ trypsin including $0.01 \%(\mathrm{w} / \mathrm{v})$ $\mathrm{CaCl}_{2}$ in $0.05 \mathrm{M}$ Tris-buffered saline ( $\mathrm{pH}$ 7.6) for $20 \mathrm{~min}$ at $37^{\circ} \mathrm{C}$. The slides were then heated in a microwave in $0.1 \mathrm{M}$ citrate buffer ( $\mathrm{pH} \mathrm{6.0)}$ at $95^{\circ} \mathrm{C}$ for $20 \mathrm{~min}$. Following antigen retrieval, endogenous peroxidase activity was quenched with $0.6 \% \mathrm{H}_{2} \mathrm{O}_{2}$ in absolute methanol for $30 \mathrm{~min}$ at room temperature. The primary antibody was applied at $4{ }^{\circ} \mathrm{C}$ overnight at the following dilutions: rabbit anti-geminin antibody (1:250), mouse anti-Ki67 antibody (1: 100; MIB-1; DAKO, Glostrup, Denmark), and rabbit anti-Mcm2 antibody (1: 500; Santa Cruz Biotechnology). Antibodies were detected using the streptavidin-biotin immunoperoxidase method using Histofine SAB reagents (Nichirei, Tokyo, Japan). Sections were then stained with 3,3-diaminobenzideine tetrahydrochloride (MP Biomedicals, Solon, OH, USA), counterstained with Meyer's hematoxylin, differenti- 
ated in water at $37^{\circ} \mathrm{C}$, dehydrated, and cleared in xylene. Incubation without the primary antibody was used as a negative control.

\section{Evaluation of geminin, Ki67, and Mcm2 labeling indices (LIs)}

To determine the LIs in each tumor, slides were evaluated in a low-power field $(\times 40)$ to identify regions with the most intense staining. Because of the uneven distribution of positive cells in the ESD specimens, all the tumor cells were counted in at least seven glands. In the advanced gastric cancers, LIs were evaluated at the tumor's invasive front, because of lower LIs in the center of the tumor. High-power fields $(\times 400)$ were captured from the selected areas using a charge-coupled device camera. Counts were performed using a Flovel Image Filing System (FlvFs; Flovel, Tachikawa, Japan). More than 700 nuclei were counted for each case. Quantitative analysis was performed by an assessor unaware of the clinicopathological variables. The LI was calculated by dividing the number of positive cells by the total number of cells counted.

\section{Double-labeling immunofluorescence}

Paraffin-embedded tissue sections were processed for double-labeling immunofluorescence, as described previously [19]. Sections were incubated with a mixture of anti-geminin (1: 100) and anti-Ki67 antibodies (1:100). They were incubated with R-phycoerythrin-conjugated goat anti-rabbit IgG (1: 200; Molecular Probes, Eugene, OR, USA) followed by Alexa Fluor 488-conjugated rabbit anti-mouse IgG (1: 200; Molecular Probes) and observed under a fluorescence microscope (Eclipse 80i; Nikon Instruments, Tokyo, Japan).

\section{Statistical analysis}

Correlations among LIs for geminin, Mcm2, and Ki67 were analyzed using Pearson's correlation coefficient test. Correlations between LIs and clinicopathological variables were analyzed using the Mann-Whitney $U$-test for the analysis of two values and the Kruskal-Wallis rank test for more than two values. Kaplan-Meier cumulative survival curves were constructed for the LIs [20]. A log-rank test was used to assess the statistical significance of the curves. Univariate and multivariate Cox regression models for survival were used to evaluate the contributions of various factors. $P<0.05$ was considered to indicate statistical significance. The analyses were carried out using Statcel ver. 2 for Windows (OMS Publishing, Saitama, Japan) and Dr. SPSS II for Windows (SPSS, Tokyo, Japan).

\section{Results}

Geminin protein expression in human gastric cancer cell lines

Geminin protein was detected at various levels in all seven gastric cancer cell lines, regardless of their histological type, as two clear bands of 25 and $28 \mathrm{kDa}$ (Fig. 1).

\section{Immunohistochemistry and double-labeling immunofluorescence}

In the 72 ESD specimens, geminin, Mcm2, and Ki67 expressions were noted exclusively in the nucleus in both normal and tumor cells, except for prophase mitotic cells, where geminin immunoreactivity was found in the cytoplasm. All protein-positive cells were observed in the neck of both non-neoplastic fundic and pyloric glands, in the lower portion of intestinal metaplasia, and in the germ center of lymphoid follicles in the lamina propria that corresponded to the proliferative zone. Geminin was localized in the nucleus, but in prophase and metaphase it was diffusely distributed in cytoplasm, and it was not shown in anaphase cells. $\mathrm{Mcm} 2$ and Ki67 were localized in the nucleus and nucleolus. In mitotic cells, Mcm2 was localized in the cytoplasm, and Ki67 was localized in the cytoplasm and chromosomes.

Double-labeling confocal immunofluorescence microscopy demonstrated that all geminin-positive cells (Fig. 2A; red) also expressed Ki67 (Fig. 2B; green), giving a yellow signal when the images were merged (Fig. 2C). No geminin-only-positive cells were detected. Similar results were found in non-neoplastic mucosa and adenomas.

Geminin- (Fig. 3A-D), Ki67- (Fig. 3 E-H), and Mcm2 (Fig. 3 I-L)-positive cells were variably located in the intramucosal proliferative lesions. In HPPs, there were a few cells expressing the three proteins in the bottom of the glands (Fig. 3A, E, I). In LGAs, geminin-positive
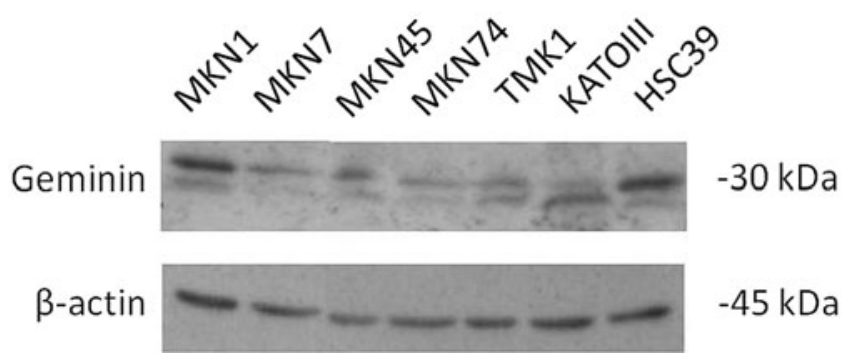

Fig. 1. Western blot analysis of geminin expression in seven human gastric cancer cell lines. $\beta$-Actin was used as an internal control. The molecular markers are indicated on the right. Geminin is noted at $25 \mathrm{kDa}$ and $28 \mathrm{kDa}$ 

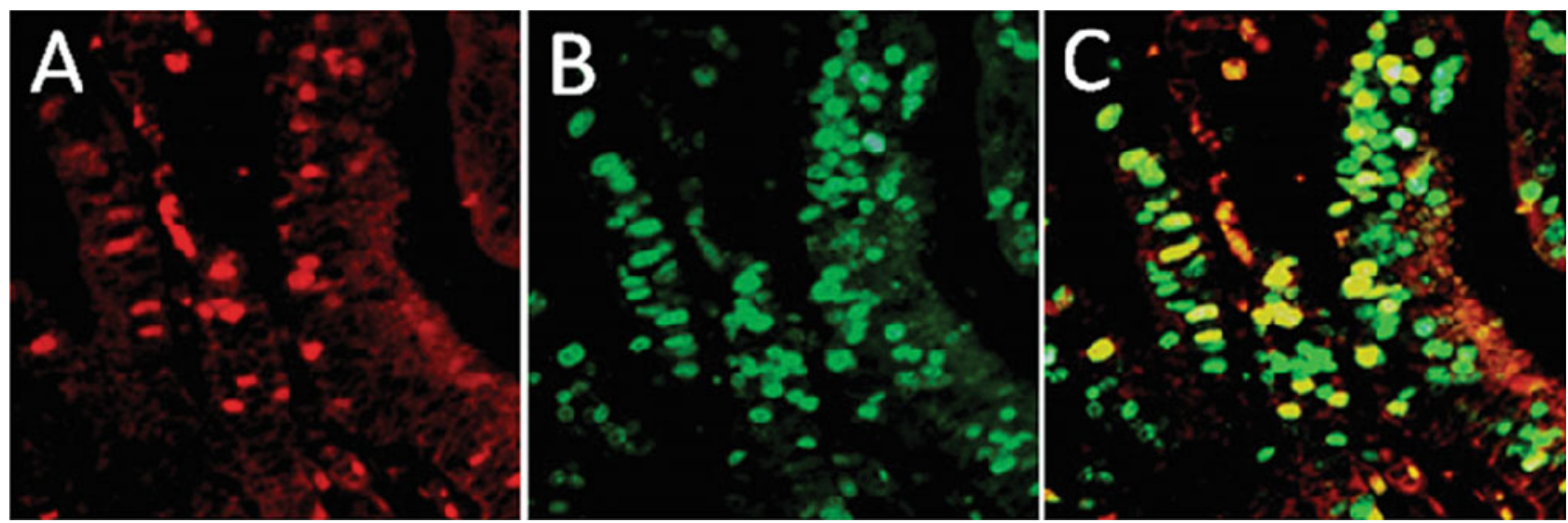

Fig. 2A-C. Double-labeling immunofluorescent staining for geminin and Ki67 in human gastric cancer specimens. Gemininpositive cells $(\mathbf{A} ;$ red $)$ coexpressed Ki67 (B; green). When the images were merged, many tumor cells showed yellow signals, indicating combined geminin and $\mathrm{Ki67}$ positivity $(\mathbf{C}) . \times 400$
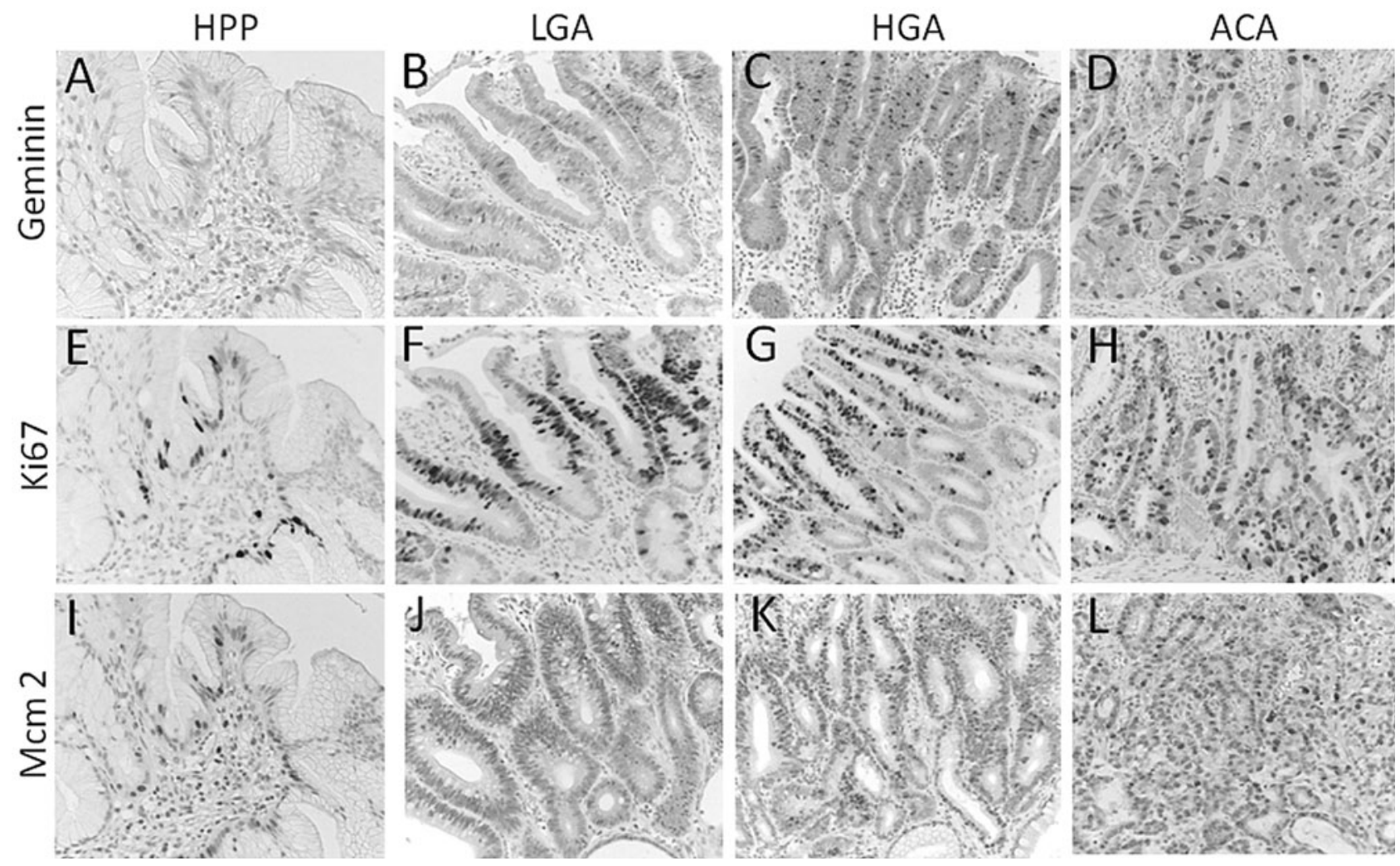

Fig. 3A-L. Immunohistochemical analysis of geminin, Ki67, and minichromosome maintenance 2 (Mcm2). Each of the fields used for analysis of geminin (A-D), Ki67 (E-H), and Mcm2 (I-L) were photographed from the same area of each lesion for hyperplastic polyps $(H P P ; \mathbf{A}, \mathbf{E}, \mathbf{I})$, low-grade adenomas $(L G A ; \mathbf{B}, \mathbf{F}, \mathbf{J})$, high-grade adenomas $(H G A ; \mathbf{C}, \mathbf{G}, \mathbf{K})$, and adenocarcinomas $(A C A ; \mathbf{D}, \mathbf{H}, \mathbf{L}) . \times 100$

cells were localized only in the middle zone of the glands (Fig. 3B), while Ki67- and Mcm2-positive cells were localized from the surface to the middle zone of the glands (Fig. 3F, J). In HGAs, protein-positive cells were located from the surface to the middle zone of the glands (Fig. 3C, G, K). In ACAs, positive cells were diffusely distributed (Fig. 3D, H, L).

The mean LIs of the proteins in the ESD lesions are shown in Table 1. When all lesion types were compared, the Mcm2 LIs were significantly higher than those for 
Table 1. Geminin, Ki67, and Mcm2 expression in endoscopic submucosal dissection lesions

\begin{tabular}{lcccc}
\hline & $n$ & Geminin & Ki67 & Mcm2 \\
\hline HPP & 14 & $3.9(3.2-6.1)$ & $17.7(17.3-19.2)$ & $26.7(22.8-38.3)$ \\
LGA & 22 & $10.5(2.7-19.9)$ & $42.2(21.0-56.0)$ & $70.0(31.6-89.8)$ \\
HGA & 10 & $18.6(8.3-26.7)$ & $52.6(33.7-74.8)$ & $67.8(47.7-79.7)$ \\
ACA & 26 & $27.2(12.9-40.9)$ & $59.7(35.9-88.4)$ & $77.8(50.0-89.9)$ \\
\hline
\end{tabular}

Data shown are mean percentages of positive cells (interquartile ranges)

Mcm2, minichromosome maintenance 2; HPP, hyperplastic polyp; LGA, low-grade adenoma;

HGA, high-grade adenoma; ACA, adenocarcinoma

Table 2. Correlation between the geminin labeling index (LI) and clinicopathological profiles

\begin{tabular}{|c|c|c|c|c|c|}
\hline & \multirow[b]{2}{*}{$n$} & \multicolumn{3}{|c|}{ Geminin } & \multirow[b]{2}{*}{$P$ value } \\
\hline & & Mean LI (\%) & LI $25 \%$ & $\mathrm{LI}>25 \%$ & \\
\hline Age (years) & & & & & 0.89 \\
\hline 70 & 65 & 24.3 & 38 & 27 & \\
\hline$>70$ & 63 & 24.8 & 35 & 28 & \\
\hline Sex & & & & & 0.17 \\
\hline Male & 100 & 24.1 & 59 & 41 & \\
\hline Female & 28 & 26.5 & 14 & 14 & \\
\hline Histological type & & & & & $<0.04$ \\
\hline pap & 14 & 28.0 & 7 & 7 & \\
\hline tub1 & 14 & 19.4 & 10 & 4 & \\
\hline tub2 & 65 & 23.2 & 38 & 27 & \\
\hline por1 & 35 & 27.0 & 18 & 17 & \\
\hline $\mathrm{T}$ stage & & & & & 0.61 \\
\hline $\mathrm{T} 2$ & 68 & 24.3 & 39 & 29 & \\
\hline $\mathrm{T} 3$ & 55 & 24.7 & 33 & 22 & \\
\hline T4 & 5 & 26.9 & 1 & 4 & \\
\hline $\mathrm{N}$ stage & & & & & $<0.02$ \\
\hline N (-) & 33 & 21.0 & 24 & 9 & \\
\hline $\mathrm{N}(+)$ & 95 & 25.8 & 49 & 46 & \\
\hline UICC stage & & & & & $<0.04$ \\
\hline I & 33 & 21.0 & 24 & 9 & \\
\hline II & 20 & 25.6 & 10 & 10 & \\
\hline III & 70 & 25.4 & 39 & 31 & \\
\hline IV & 5 & 32.6 & 0 & 5 & \\
\hline Mcm2 LI & & & & & $<0.0001$ \\
\hline 65.0 & 60 & 20.4 & 48 & 12 & \\
\hline$>65.0$ & 68 & 28.3 & 25 & 43 & \\
\hline Ki67 LI & & & & & $<0.0001$ \\
\hline 45.0 & 63 & 20.0 & 48 & 15 & \\
\hline$>45.0$ & 65 & 29.0 & 25 & 40 & \\
\hline
\end{tabular}

tub1, well-differentiated type; tub2, moderately differentiated; por1, poorly differentiated and solid type; UICC, International Union Against Cancer

Ki67, which were significantly higher than those for geminin $(P<0.0001)$. The geminin LIs of HPPs, LGAs, HGAs, and ACAs were $3.9 \%, 10.5 \%, 18.6 \%$, and $27.2 \%$, respectively $(P<0.001)$. The Ki67 LI in HPPs was $17.7 \%$, which was significantly lower than that in LGAs (42.2\%), HGAs (52.6\%), and ACAs (59.7\%). The Ki67 LI in LGAs was significantly lower than those in HGAs and ACAs. The Mcm2 LI in HPPs (26.7\%) was also significantly lower than those in all the other lesions (LGA; 70.0\%, HGA; 67.8\%, ACA; 77.8\%) $(P<0.001)$.
Correlations between geminin LI and clinicopathological profile in advanced gastric cancer

The mean LIs for Mcm2, Ki67, and geminin were $64.3 \%$ (median, 66.1\%), 47.4\% (median, $45.5 \%$ ), and $24.6 \%$ (median, 23.7\%), respectively, in the 128 advanced gastric cancers. Table 2 shows the relationship between geminin LIs and clinicopathological profiles. We chose LI cutoff values for geminin $(25 \%), \mathrm{Mcm} 2(65 \%)$, and Ki67 (45\%), the values being almost equal to the mean/ 
Table 3. Univariate and multivariate Cox regression analyses for predictors of overall survival in patients with advanced intestinal-type gastric carcinoma

\begin{tabular}{lllcr}
\hline & RR & 95\% Confidence interval & $P$ \\
\hline Univariate analysis & & & $(1.47-15.57)$ & 0.01 \\
N (+) & vs N (-) & 4.79 & $(1.01-4.05)$ & 0.05 \\
ly1 & vs ly0 & 3.02 & $(1.55-16.70)$ & 0.01 \\
UICC stage III & vs Stage I & 5.08 & $(1.05-3.59)$ & 0.04 \\
Geminin (>25\%) & vs (25\%) & 1.94 & $(0.63-2.16)$ & 0.62 \\
Mcm2 (>65\%) & vs (65\%) & 1.17 & $(0.54-1.85)$ & 1.00 \\
Ki67 (>45\%) & vs (45\%) & 1.00 & $(1.36-14.53)$ & 0.02 \\
Multivariate analysis & vs N (-) & 4.46 & & \\
N (+) & & &
\end{tabular}

median LIs. We divided the patients into two groups with LIs either above (high) or below (low) the cutoff values. High LIs for geminin, $\mathrm{Mcm} 2$, and Ki67 were noted in 55, 68, and 65 patients, respectively. The LIs for geminin were significantly higher in papillary and solidtype adenocarcinomas than in well- and moderately differentiated tubular adenocarcinomas. The LIs for geminin were significantly correlated with $\mathrm{N}$ stage $(P<$ $0.02)$, UICC stage $(P<0.04)$, high Mcm2 LIs $(>65 \% ; P$ $<0.0001)$, and high Ki67 LIs ( $>45 \% ; P<0.0001)$, but not with age, sex, or T stage.

We examined the cumulative survival of the high and low LI groups using the Kaplan-Meier method and log-rank tests. There were no significant correlations between geminin LIs and survival for stages I and stage II $(P<0.54$ and $P<0.73$, respectively; Fig. 4A,B). However, high geminin LIs were associated with poorer survival in the 70 stage III patients $(P<0.05$; Fig. $4 \mathrm{C})$. Moreover, the 55 patients in stages I-IV with high geminin LIs had significantly poorer prognoses than the 73 patients in stages I-IV with low geminin LIs $(P<0.04$; Fig. 4D). Mcm2 and Ki67 LIs were not correlated with survival in stages I-IV (Fig. 4E, F), and in stage III $(P>0.93$ and $P>0.57$, respectively) (figure not shown).

Univariate Cox regression analysis indicated that the overall survival of stage I-IV tumors was significantly correlated with high geminin LIs (relative risk [RR] = 1.94; $P=0.04$; Table 3). Multivariate Cox regression analysis was used to determine the prognostic value of the markers, age, sex, histological differentiation, $\mathrm{T}$ stage, $\mathrm{N}$ status, and UICC stage. $\mathrm{N}$ status was identified as an independent prognostic factor.

\section{Discussion}

The estimated molecular weight of geminin protein is $25 \mathrm{kDa}$, but the immunoblotting of proteins from seven gastric cancer cell lines in the present study produced two bands, of 25 and $28 \mathrm{kDa}$. The oncosup- pressor, retinoblastoma protein, also shows multiple bands on western blots [21], and this is due to the phosphorylation of serine/threonine sites by cdc2 kinase. Geminin is thought to be phosphorylated at both serine/threonine and tyrosine residues by cell cycledependent kinases [22,23] and it is therefore possible that the $28-\mathrm{kDa}$ geminin band could have resulted from hyperphosphorylation.

Geminin blocks re-replication of the genome in the same cell cycle. It is present during the $\mathrm{S} / \mathrm{G} 2 / \mathrm{M}$ phases of the cell cycle but absent during G1, and thus acts as an $\mathrm{S} / \mathrm{G} 2 / \mathrm{M}$ marker. Mcm2-positive cells are thought to occur not only during cell cycling, but also during the G0 phase, or "dormancy" [12]. Ki67 is expressed during all phases of the cell cycle, except for G0 [24], and may be present during cell-cycle arrest. In accordance with these features, we found that the geminin LI was significantly lower than that of Ki67, and the latter was lower than the Mcm2 LIs in both normal and tumor cells. In the present study, the LIs of the three markers in HPP, LGA, HGA, and ACA were: geminin, $3.9 \%, 10.5 \%$, $18.6 \%$, and $27.2 \%$; Ki67, $17.7 \%, 42.2 \%, 52.6 \%$, and $59.7 \%$; Mcm $2,26.7 \%, 70.0 \%, 67.8 \%$, and $77.8 \%$. These findings are consistent with the published data $[12,19]$.

The low Mcm2 LIs could be explained if most HPP cells were differentiated or out of the cell cycle, while LGAs could have more G0 phase or dormant cells with potential proliferative activity (high $\mathrm{Mcm} 2$ and low Ki67 and geminin LIs). HGAs could include more arrested G1 cells (high Mcm2 and Ki67, and low geminin LIs), which would be difficult to differentiate from ACA. ACAs showed the highest geminin LI among the intramucosal neoplasms, indicative of high proliferative activity. The ratio of geminin/Ki67 represents the percentage of active cycling cells in all the proliferating cells. In the present study, the ratio of ACA (0.46) was definitely higher than those of HPP (0.22), LGA (0.19), and HGA (0.33).

Our results suggest that geminin could reflect the population of "active" cycling cells in S/G2/M phase in neoplastic lesions. Cho et al. [25] studied the cell kinetics 

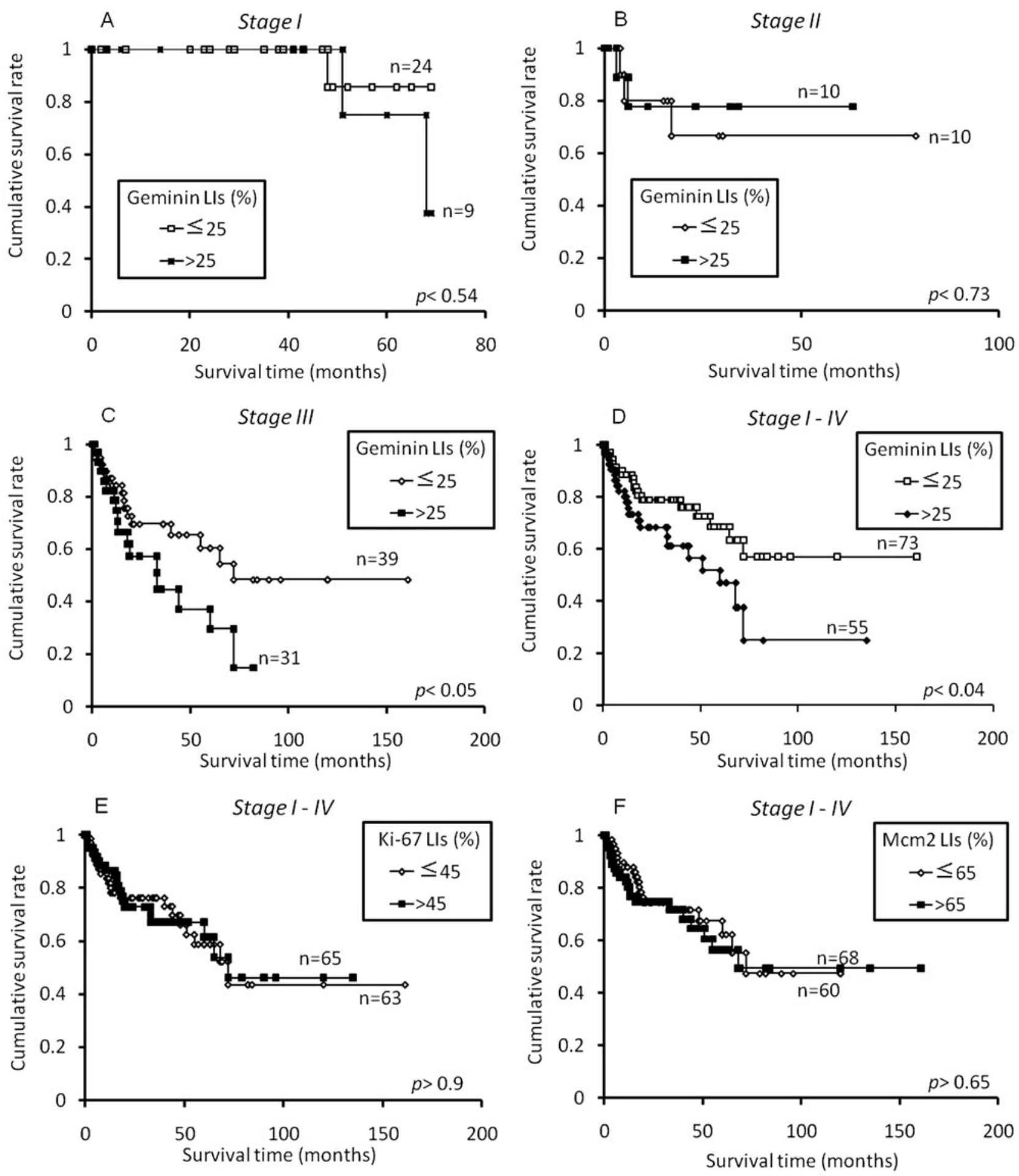

Fig. 4A-F. Kaplan-Meier curves showing cumulative overall survival rates according to the markers. Cutoff values were chosen at $25 \%$ for geminin, $45 \%$ for Ki67, and $65 \%$ for $\mathrm{Mcm} 2$. In stage I and stage II patients, a higher geminin labeling index $(L I ;>25 \%)$ did not correlate with poorer survival (A and $\mathbf{B}$; $P<0.54$ and $P<0.73$, respectively). The cumulative survival of the 55 patients in stage I-IV with a high geminin LI (>25\%)

was significantly poorer than that of the 73 patients in stage I-IV with a low LI $(\leq 25 \%)(P<0.04 ; \mathbf{D})$. Patients in stage III with a high geminin LI showed poorer survival $(P<0.05$; $\mathbf{C})$. High Ki67 LI (>45\%) and Mcm2 LI (>65\%) in stage I-IV patients did not correlate with poorer survival $(\mathbf{E}$ and $\mathbf{F} ; P>$ 0.9 and $P>0.65$, respectively) 
of proliferative gastric lesions and found that human growth and transformation-dependent protein expression occurred during the progression from normal gastric mucosa to intestinal-type carcinoma and might be associated with tumor cell proliferation. The present study suggests that the assessment of cell kinetics using anti-geminin, Ki67, and $\mathrm{Mcm} 2$ antibodies might permit differentiation among HPPs, LGAs, HGAs, and ACAs. Quaglia et al. [26] reported that the combination of Mcm2, geminin, and Ki67 could represent a valuable tool in differentiating hepatocellular carcinoma from regenerative and dysplastic nodules in liver cirrhosis. Montanari et al. [14] suggested that increased expression of geminin promoted cell-cycle progression and proliferation in normal and cancerous breast cells in vitro, suggesting that higher geminin LIs could be causally associated with the early stages of gastric carcinogenesis. These results are consistent with our data, which showed higher geminin, Ki67, and Mcm2 LIs in intramucosal gastric cancer than in advanced gastric cancers.

Higher geminin LIs were associated with more proliferative tumors and poorer prognoses. We previously demonstrated the prognostic significance of geminin in human colorectal and oral cancers [19,27]. Other studies have reported the potential prognostic significance of geminin in breast, kidney, brain, and oral tumors $[15,12$, 28, 29], in line with our results.

We recently demonstrated the prognostic value of a combination of $\mathrm{Mcm} 2$ and Ki67 for the assessment of stage III diffuse-type gastric cancer, but not the intestinal type [30]. Thus, the addition of geminin increased the predictive prognostic value of Mcm2 and Ki67.

Previous reports found that geminin expression was a poor prognostic marker of overall survival in patients with breast, kidney, and colorectal cancers [15, 12, 19]. However, Shrestha et al. [28] reported that higher geminin LIs might be associated with longer survival in patients with high-grade astrocytic tumors with preoperative chemotherapy. We also found that higher geminin LIs were associated with better prognoses in patients with stage III-IV oral squamous cell carcinoma receiving preoperative radiochemotherapy [27]. These conflicting results could be explained by an increase in radiochemosensitivity associated with geminin overexpression; some studies have shown that cells synchronized in G1 phase were more radioresistant than cells in the S, late $\mathrm{G} 2$, and $\mathrm{M}$ phases [31,32].

There are some limitations of the present study. We omitted diffuse-type gastric cancers from advanced gastric cancers because of difficulties in counting the poorly cohesive tumor cells mixed with fibroblasts and inflammatory cells. Second, it is possible that there were too few cases to demonstrate the relationship between cell kinetics, including those of geminin, and patient outcomes in the multivariate Cox regression analysis.
Many studies have evaluated the relationships between advanced gastric cancer prognosis and known proliferative markers, such as Ki67. Some studies found that high Ki67 LIs were correlated with poorer survival [33, 34], whereas others did not [35]. In our previous study, $\mathrm{Mcm} 2$ and Ki67 expression did not correlate with any clinicopathological factors, e.g., lymph node status or vascular invasion in stage III intestinal-type gastric cancers [30], which is consistent with our present data. Although both $\mathrm{Mcm} 2$ and Ki67 occur in active cycling cells, it is possible that they also occur in slow/arrested $\mathrm{G} 1$, and Mcm2 is expressed even in G0 phase [36, 37]. These markers may not therefore reflect the proliferative activity of tumor cells and may not be suitable predictive markers in patients with malignant tumors.

In conclusion, geminin expression might facilitate the proliferation of intramucosal neoplasms of the stomach. The geminin LI could serve as a marker for active cycling tumor cells and have prognostic significance in intestinal-type gastric carcinomas. The role of geminin might also have significant implications for the development and application of targeted therapies.

Acknowledgments We thank Mr. N. Itaki, Ms. M. Iwatani, and Ms. C. Yamasaki (Division of Organ Pathology, Faculty of Medicine, Tottori University) for their skillful technical assistance and Ms. Y. Tokuoka for assistance with collecting patient information. This work was supported in part by a Tottori University Faculty of Medicine Research Grant for 2008 (No. 310530).

\section{References}

1. Kamangar F, Dores GM, Anderson WF. Patterns of cancer incidence, mortality, and prevalence across five continents: defining priorities to reduce cancer disparities in different geographic regions of the world. J Clin Oncol 2006;24:2137-50.

2. Cunningham D, Allum WH, Stenning SP, Thompson JN, Van de Velde CJ, Nicolson M, et al. Perioperative chemotherapy versus surgery alone for resectable gastroesophageal cancer. N Engl J Med 2006;355:11-20.

3. Ajani JA, Moiseyenko VM, Tjulandin S, Majlis A, Constenla M, Boni C, et al. Quality of life with docetaxel plus cisplatin and fluorouracil compared with cisplatin and fluorouracil from a phase III trial for advanced gastric or gastroesophageal adenocarcinoma: The V-325 study group. J Clin Oncol 2007;25:3210-6.

4. Fenoglio-Preiser C, Carneiro F, Correa P, Guilford P, Lambert R, Megraud F, et al. Gastric carcinoma. In: Hamilton SR, Aaltonen LA, editors. World Health Organization classification of tumours. Pathology and genetics of tumours of the digestive system. Lyon, France: IARC; 2000. p. 47-8.

5. Kamiya T, Morishita T, Asakura H, Miura S, Munakata Y, Tsuchiya M. Long-term follow-up study on gastric adenoma and its relation to gastric protruded carcinoma. Cancer 1982;50:2496-503.

6. Cristallini EG, Ascani S, Bolis GB. Association between histologic type of polyp and carcinoma in the stomach. Gastrointest Endosc 1992;38:481-4.

7. Lee JH, Abraham SC, Kim HS, Nam JH, Choi C, Lee MC, et al. Inverse relationship between APC gene mutation in gastric ade- 
nomas and development of adenocarcinoma. Am J Pathol 2002; 161:611-8.

8. Misdraji J, Lauwers GY. Gastric epithelial dysplasia. Semin Diagn Pathol 2002;19:20-30.

9. McGarry TJ, Kirschner MW. Geminin, an inhibitor of DNA replication, is degraded during mitosis. Cell 1998;93:1043-53.

10. Wohlschlegel JA, Dwyer BT, Dhar SK, Cvetic C, Walter JC, Dutta A. Inhibition of eukaryotic DNA replication by geminin binding to Cdt1. Science 2000;290:2309-12.

11. Nishitani H, Lygerou Z. Control of DNA replication licensing in a cell cycle. Genes Cells 2002;7:523-34.

12. Wohlschlegel JA, Kutok JL, Weng AP, Dutta A. Expression of geminin as a marker of cell proliferation in normal tissues and malignancies. Am J Pathol 2002;161:267-73.

13. Dudderidge TJ, Stoeber K, Loddo M, Atkinson G, Fanshawe T, Griffiths DF, et al. Mcm2, geminin, and Ki67 define proliferative state and are prognostic markers in renal cell carcinoma. Clin Cancer Res 2005;11:2510-7.

14. Montanari M, Boninsegna A, Faraglia B, Coco C, Giordano A, Cittadini A, et al. Increased expression of geminin stimulates the growth of mammary epithelial cells and is a frequent event in human tumors. J Cell Physiol 2005;202:215-22.

15. Shetty A, Loddo M, Fanshawe T, Prevost AT, Sainsbury R, Williams GH, et al. DNA replication licensing and cell cycle kinetics of normal and neoplastic breast. Br J Cancer 2005;93: 1295-300.

16. Gonzalez MA, Tachibana KE, Chin SF, Callagy G, Madine MA, Vowler SL, et al. Geminin predicts adverse clinical outcome in breast cancer by reflecting cell-cycle progression. J Pathol 2004; 204:121-30.

17. Lauren $\mathrm{P}$. The two histological main types of gastric carcinoma. Diffuse and so-called intestinal-type carcinoma. Acta Pathol Microbiol Scand 1965;64:31-49.

18. Japanese Gastric Cancer Association, editors. The Japanese classification of gastric carcinoma. 14th ed. Tokyo: Kanehara; 2010. p. 8-17.

19. Nishihara K, Shomori K, Tamura T, Fujioka S, Ogawa T, Ito H. Immunohistochemical expression of geminin in colorectal cancer: implication of prognostic significance. Oncol Rep 2009;21: 1189-95.

20. Kaplan EL, Meier P. Nonparametric estimation for incomplete observation. J Am Stat Assoc 1958;53:457-81.

21. Lin BT, Gruenwald S, Morla AO, Lee WH, Wang JY. Retinoblastoma cancer suppressor gene product is a substrate of the cell cycle regulator cdc2 kinase. EMBO J 1991;10:857-64.

22. Kulartz M, Kreitz S, Hiller E, Damoc EC, Przybylski M, Knippers R. Expression and phosphorylation of the replication regulator protein geminin. Biochem Biophys Res Commun 2003;305: 412-20.

23. Nakuci E, Xu M, Pujana MA, Valls J, Elshamy WM. Geminin is bound to chromatin in $\mathrm{G} 2 / \mathrm{M}$ phase to promote proper cytokinesis. Int J Biochem Cell Biol 2006;38:1207-20.
24. Duchrow M, Ziemann T, Windhövel U, Bruch HP, Broll R. Colorectal carcinomas with high MIB-1 labelling indices but low pKi67 mRNA levels correlate with better prognostic outcome. Histopathology 2003;42:566-74.

25. Cho YE, Kim JY, Kim YW, Park JH, Lee S. Expression and prognostic significance of human growth and transformationdependent protein in gastric carcinoma and gastric adenoma. Hum Pathol 2009;40:975-81.

26. Quaglia A, McStay M, Stoeber K, Loddo M, Caplin M, Fanshawe $\mathrm{T}$, et al. Novel markers of cell kinetics to evaluate progression from cirrhosis to hepatocellular carcinoma. Liver Int 2006;26: 424-32.

27. Tamura T, Shomori K, Haruki T, Nosaka K, Hamamoto Y, Shiomi $\mathrm{T}$, et all. Minichromosome maintenance-7 and geminin are reliable prognostic markers in patients with oral squamous cell carcinoma: immunohistochemical study. J Oral Pathol Med 2010; 39:328-34.

28. Shrestha P, Saito T, Hama S, Arifin MT, Kajiwara Y, Yamasaki F, et al. Geminin: a good prognostic factor in high-grade astrocytic brain tumors. Cancer 2007;109:949-56.

29. Loddo M, Kingsbury SR, Rashid M, Proctor I, Holt C, Young J, et al. Cell-cycle-phase progression analysis identifies unique phenotypes of major prognostic and predictive significance in breast cancer. Br J Cancer 2009;100:959-70.

30. Tokuyasu N, Shomori K, Nishihara K, Kawaguchi H, Fujioka S, Yamaga $\mathrm{K}$, et al. Minichromosome maintenance 2 (MCM2) immunoreactivity in stage III human gastric carcinoma: clinicopathological significance. Gastric Cancer 2008;11:37-46.

31. Hama S, Matsuura S, Tauchi H, Yamasaki F, Kajiwara Y, Arita K, et al. Gene transfer increases cell killing with abnormal nucleation after ionising radiation in glioma cells. Br J Cancer 2003;89: 1802-11.

32. Terasima T, Tolmach LJ. Variations in several responses of HeLa cells to x-irradiation during the division cycle. Biophys J 1963;3: 11-33.

33. Yonemura Y, Ohoyama S, Kimura H, Kamata T, Yamaguchi A, Miyazaki I. Assessment of tumor cell kinetics by monoclonal antibody Ki-67. Eur Surg Res 1990;22:365-70.

34. Chen L, Li X, Wang GL, Wang Y, Zhu YY, Zhu J. Clinicopathological significance of overexpression of TSPAN1, Ki67 and CD34 in gastric carcinoma. Tumori 2008;94:531-8.

35. Oshima CT, Iriya K, Forones NM. Ki-67 as a prognostic marker in colorectal cancer but not in gastric cancer. Neoplasma 2005; $52: 420-4$.

36. Stoeber K, Tlsty TD, Happerfield L, Thomas GA, Romanov S, Bobrow L, et al. DNA replication licensing and human cell proliferation. J Cell Sci 2001;114:2027-41.

37. Gerdes J, Lemke H, Haisch H, Wacker HH, Schwab U, Stein H. Cell cycle analysis of a cell proliferation-associated human nuclear antigen defined by the monoclonal antibody Ki-67. J Immunol $1984 ; 133: 1710-5$ 